\title{
INVESTIGACIONES
}

\section{Educación familiar e intercultural en contexto mapuche: hacia una articulación educativa en perspectiva decolonial ${ }^{*}$}

\author{
Family and intercultural education in Mapuche context: \\ toward an educative articulation in decolonial perspective \\ Gerardo Muñoz Troncoso ${ }^{a, b, c, d}$ \\ ${ }^{a}$ Instituto de Ciencias de la Educación, Universidad Austral de Chile. \\ gerardo.munoz01@uach.cl \\ ${ }^{b}$ Centro de Investigación en Educación en Contexto Indígena e Intercultural (CIECII), \\ Universidad Católica de Temuco. \\ ${ }^{c}$ Centro de Investigación en Educación para la Justicia Social (CIEJUS), \\ Universidad Católica del Maule. \\ ${ }^{d}$ Grupo ERDISC, Universidad Autónoma de Barcelona.
}

\begin{abstract}
RESUMEN
El manuscrito busca problematizar la implementación de la educación intercultural en Chile y su carácter funcionalista, como base para la construcción de un proyecto decolonial de educación intercultural en contexto mapuche. A través de una revisión literaria, se analiza la educación familiar mapuche, la educación escolar, las discontinuidades que entre ambas se establecen y cómo el carácter funcionalista de la educación intercultural propició la continuidad del proyecto colonial y el monoculturalismo del Estado en áreas mapuches. Ante esta situación, se propone la necesidad de avanzar en la construcción de un proyecto decolonial de educación intercultural que propicie la articulación entre la educación familiar y escolar. Finalmente, se sostiene que, dicho proyecto decolonial es factible al asumir un pluralismo epistemológico que sustente una nueva forma de comprender la educación en contexto indígena, que propicie el pleno desarrollo socio-educativo de las personas y abandone su labor asimilacionista.
\end{abstract}

Palabras claves: educación familiar mapuche, educación intercultural, discontinuidades educativas, decolonialismo, pluralismo epistemológico.

\begin{abstract}
The manuscript seeks to problematize the implementation of intercultural education in Chile, and its functionalist character as a basis for the construction of a decolonial project of intercultural education in the Mapuche context. Through a literary review, the Mapuche family education, school education, the discontinuities established between them and how the functionalist nature of intercultural education led to the continuity of the colonial project and the monoculturalism of the State in Mapuche areas are analyzed. Given this situation, the need to move forward in the construction of a decolonial intercultural education project, that fosters the articulation between family and school education, is proposed. Finally, it is argued that said decolonial project is feasible by assuming an epistemological pluralism that supports a new way of understanding education in an indigenous context, that favours the full socio-educational development of people and abandons their assimilationist work.

Key words: Mapuche family education, intercultural education, educational discontinuities, decolonialism, epistemological pluralism.
\end{abstract}

\footnotetext{
* Agradecimientos al Proyecto del Fondo Nacional de Desarrollo Científico y Tecnológico (FONDECYT) Iniciación $\mathrm{N}^{\mathrm{o}}$ 11191041 y al Proyecto FONDECYT Regular 1181531.
} 
Estudios Pedagógicos XLVII N 1: 391-407, 2021

EDUCACIÓN FAMILIAR E INTERCULTURAL EN CONTEXTO MAPUCHE: HACIA UNA ARTICULACIÓN EDUCATIVA EN PERSPECTIVA DECOLONIAL

\section{INTRODUCCIÓN}

En Chile, desde la política pública se ha construido un discurso que posiciona la educación escolar como una oportunidad y motor de movilidad social. Con este propósito, en las últimas décadas se han implementado un conjunto de reformas, que abordan tanto lo curricular, como el sitema de financiamiento de los centros educativos, con el propósito de establecer las bases para el mejoramiento de la calidad de la educación ofrecida (Paredes, 2016). Sin embargo, la educación escolar implementada ha prescindido de su vinculación con los actores del medio familiar y comunitario del cual proceden los estudiantes, desconociendo el valor de estos y sus formas de conocer (Muñoz, Quintriqueo y Essomba, 2019), al igual que en los inicios de la educación pública chilena, en la primera mitad del s. XIX (Serrano, Ponce y Rengifo, 2013a). Lo anterior, en contexto indígena y mapuche en particular, propició el establecimiento una discontinuidad entre la educación familiar y la educación escolar, donde se observa que los estudiantes de ascendencia mapuche abandonan progresivamente los saberes y conocimientos educativos propios, producto de una enseñanza escolar descontextualizada a su realidad sociocultural (Muñoz, 2016). Esta discontinuidad es el resultado de una educación de carácter colonial, monocultural eurocéntrico y racista en La Araucanía, lo que se constituye en la base de los conflictos y tensiones que construyen la identidad sociocultural de las nuevas generaciones de mapuches (Mansilla, Llancavil, Mieres y Montanares, 2016).

Desde esa perspectiva histórica, la escolarización implementada en contexto mapuche se ha desarrollado mediante la evangelización (s. XVI), las escuelas misionales (s. XVIII), la escuela pública y los internados (s. XIX), los que coexisten hasta la actualidad (Muñoz y Quintriqueo, 2019). Estos modelos han incidido directamente en la discontinuidad educativa en contexto mapuche, al ser el medio de ejecución del proyecto social y político colonialista del Estado chileno, que ha buscado establecer la dominación desde los marcos sociales y epistémicos de la sociedad de origen europea occidental (Mansilla, Ponce De León y Turra, 2018). Es así como la educación escolar se ha centrado en la enseñanza de la fe cristiana, el sometimiento a la sociedad hegemónica, la castellanización y el aprendizaje de la identidad nacional, desde una concepción racista en la construcción de la sociedad chilena (Lagos, 1908; Noggler, 1972; Serrano et al., 2013a; Gysling, 2015). Por consiguiente, mediante la discontinuidad educativa se ha excluido a las familias, ha negado la identidad sociocultural, ha prohibido el uso del mapunzugun -lengua mapuche- y ha construido un desprecio hacia el conocimiento mapuche en el medio escolar (Muñoz, Quintriqueo y Essomba, 2019). En este proceso, la escuela y la educación escolar han construido la colonialidad del ser, del saber y del poder en la racionalidad de las personas mapuches, para su asimilación a la sociedad chilena, mediante un modelo de inclusión social básica, como obreros o campesinos empobrecidos (Quintriqueo y Muñoz, 2015; Whitehead, 2017).

Con el fin de revertir la discontinuidad de la educación familiar y los perjuicios del tipo de escolarización desarrollada en contexto indígena, tanto en Latinoamérica como en Chile, se ha implementado la educación intercultural bilingüe (EIB). La EIB responde a una demanda de los pueblos indígenas de respecto de una educación escolar contextualizada, que incorpore la lengua, contenidos, agentes y métodos de la educación familiar, estando asociada a procesos de reivindicación social y territorial (Quilaqueo, Quintriqueo, Torres y Muñoz, 2014; Quintriqueo, Quilaqueo, Peña-Cortés y Muñoz, 2015). Es así como, desde la política pública se busca valorizar la diversidad sociocultural sobre la que se ha construido 
la sociedad chilena, aportando al reconocimiento de los pueblos indígenas que habitan el territorio nacional (Programa de Educación Intercultural Bilingüe [PEIB], 2017). En este contexto, se concuerda con Essomba (2007) al comprender la diversidad como una característica propia de la humanidad, que aporta riqueza y complejidad a las relaciones sociales, producto de las relaciones de poder, los imaginarios sociales, los valores y nociones de mundo que caracterizan a los distintos grupos humanos. Igualmente, en reconocer la interculturalidad como un modelo de abordaje de la diversidad sociocultural, en el que se busca establecer condiciones equitativas para el diálogo entre las sociedades y culturas en contacto. Por lo anterior, la apuesta del Estado chileno por la EIB en los territorios indígenas es, en origen, una decisión acertada desde sus fundamentos teóricos y en la búsqueda de justicia para los pueblos indígenas en general y mapuche en particular.

Sin embargo, estudios sobre la implementación de la EIB han evidenciado que es un modelo de educación dirigido principalmente a estudiantes indígenas, posicionándose como una educación de baja calidad (Viña, Tapia y Walsh, 2010). En el caso mapuche en Chile, se ha constatado el fracaso sistemático de la EIB, al ser desarrollado en la estructura epistémica del conocimiento occidental, siendo la continuidad de las políticas coloniales y asimilacionista del Estado chileno, en el marco de un sistema escolar neoliberalizado (Webb y Radcliffe, 2013; Luna, 2015). Lo anterior se relaciona con la permanencia de las prácticas de exclusión de la familia y comunidad mapuche del medio escolar, la inclusión parcializada y 'estandarizada' de contenidos educativos mapuches en la EIB (Muñoz, 2016) y el desconocimiento de la episteme propia en que se sustenta la educación familiar (Quintriqueo y Torres, 2012). De esta forma la discontinuidad de la educación familiar mapuche limita la intervención educativa intercultural y la colaboración para la construcción de aprendizajes contextualizados y pertinentes.

El objetivo del manuscrito es problematizar la implementación de la educación intercultural en Chile y su carácter funcionalista, como base para la construcción de un proyecto decolonial de educación intercultural en contexto mapuche.

\section{EDUCACIÓN FAMILIAR MAPUCHE}

La educación familiar mapuche es un proceso de formación de las nuevas generaciones, con el objeto transmitir la memoria individual y social de la comunidad respecto a saberes y conocimientos educativos (Quintriqueo et al., 2015). En la perspectiva de Manquilef (1914) y Coña (2017), busca la construcción de los aprendizajes mediante el desarrollo de experiencias cotidianas, en instancias formales e informales del grupo familiar. Su implementación se estructura sobre la relación de principios, contenidos, métodos y agentes educativos para la formación de las nuevas generaciones. Los principios educativos buscan formar actitudes socialmente valoradas, para que las personas tengan arraigado en sus vidas una actitud y conducta respetuosa con las demás personas (Muñoz, 2016). En la perspectiva de Quilaqueo y Quintriqueo (2017), estos principios son kümeyawael ta che (conducta de respeto), yamüwael ta che (estima y respeto), küme che geael (generosidad y bondad), küme rakizuam ta nieael (reflexividad y razonamiento) y kim che geael (ser sabio). Los contenidos educativos, son un conjunto de saberes y conocimientos que han sido construidos en la relación del hombre con el medio natural, social, cultural y espiritual, en una relación subjetiva e intersubjetiva entre los miembros del grupo familiar 
y comunitario (Quintriqueo et al., 2015; Muñoz et al., 2019). Para Coña (2017), los contenidos educativos contienen los aspectos centrales de la cosmovisión, la espiritualidad y las representaciones sobre los hechos históricos que, como sociedad han experienciado y que son determinantes para comprender el contexto de vida actual. Los métodos educativos, se vinculan principalmente a discursos educativos desarrollados al interior de la familia y comunidad, identificándose el gübam (consejo para la formación de personas), wewpin (confrontación de ideas, saberes y conocimientos entre sabios), pentukun (conversación formal para estrechar lazos sociales), ülkantun (lírica que combina la expresión poética y el canto) y piam (relatos fundacionales centrados en el valor de la espiritualidad y el ecosistema) (Quilaqueo, Fernández y Quintriqueo, 2017). Los agentes educativos son las personas que implementan la educación familiar. Al respecto, señalan Quilaqueo et al. (2017) que, si bien en este proceso participa la familia ampliada, se reconoce la existencia de personas con la capacidad de recordar y transmitir el patrimonio cultural mapuche, como el logko (principal autoridad política), wewpife (orador que desarrolla el wewpin), genpin (que desarrolla las rogativas a la fuerza superior), machi (especialista en conocimiento medicinal) y werken (mensajero), donde el uso y dominio del mapunzugun es de vital importancia.

Estudios recientes han permitido constatar que, en mapunzugun, la educación familiar se denomina kimeltuwün, que corresponde a un concepto compuesto en una lógica de aglutinación donde: 1) kim es saber y conocimiento propio; 2) el es un indicativo de orden fundado por la fuerza espiritual superior; 3) mel es estructurar racionalmente una idea; 4) tu es la base sobre la que se sostiene algo o alguien; y 5) wün es la luz, un espacio y tiempo sustentado en la lógica de la reciprocidad en el acto de enseñar y aprender (Muñoz, 2016; Quilaqueo y Quintriqueo, 2017). Por lo tanto, el kimeltuwün es un proceso social, reflexivo y espiritual en el que se sustenta la formación de las nuevas generaciones desde la lógica del conocimiento mapuche y la memoria social e histórica.

Para Quilaqueo y Quintriqueo (2017), la educación familiar mapuche se sustenta en: 1) una base epistémica propia, que permite la construcción de conocimientos educativos, sociales, culturales y espirituales; y 2) una relación que los individuos establecen con el saber desde un discurso social y cultural que sustenta la construcción de conocimientos. Igualmente, es un proceso contextual y con criterio de temporalidad, por lo que está en constante construcción y reconstrucción al interior de la familia y comunidad (Manquilef, 1914; Coña, 2017). En este contexto, se reconoce la influencia de las sociedades inca, hispana y chilena, producto de los procesos de invasión, conquista y colonización implementados desde el s. XV a la fecha (Quilaqueo, 2018). Al respecto, precisa Coña (2017) que las familias reconocen como principales instituciones que han impulsado un cambio social, la iglesia y la escuela, las que propiciaron el surgimiento de un nuevo modelo de persona, referido al mapuche escolarizado y cristiano, que transita entre la tradición mapuche y el conocimiento occidental. No obstante, la incorporación de componentes de la cultura chilena-occidental se realizó empleando el control cultural, para su utilización desde la propia lógica del conocimiento mapuche (Bonfil, 1988; Quilaqueo, 2005). Por consiguiente, la educación familiar mapuche es un proceso complejo y sistemático, cuyo fin es formar a las nuevas generaciones desde el saber y conocimiento propio, en función del contexto social y temporal en que deberán desarrollar sus vidas. En la investigación se sostiene que la incorporación del kimeltuwün a la educación escolar desde un enfoque educativo intercultural, en tanto acción educativa propia a la episteme mapuche, contribuiría significativamente en revertir la discontinuidad educativa de estudiantes mapuches. 


\section{ESCOLARIZACIÓN EN CONTEXTO MAPUCHE}

La escolarización se refiere a un recorrido de formación donde las personas aprenden un conjunto de contenidos, actitudes, reglas, normas y creencias, sea de forma planificada o no, formal e informal, en el marco de las institucionalidades definidas por la sociedad hegemónica (Ander-Egg, 2014). Señala Muñoz et al. (2019), que en contexto mapuche ha sido implementada tanto por el Imperio español (1553-1810), como por el Estado chileno (1810 al presente), mediante la evangelización, las escuelas misionales, las escuelas públicas y los internados. La evangelización se instaló con la llegada de los conquistadores españoles como un proceso de transmisión de valores, orden moral, social y religioso eurocéntrico occidental, con la finalidad de transformar a niños y jóvenes mapuches en un ser cristiano, enajenado de su propia cultura y sometido al Imperio español en obediencia a Dios (Noggler, 1972). La escuela misional surge con la creación de los Colegios de Propaganda Fide en Europa, correspondientes a seminarios teológicos para la formación de misioneros (Lagos, 1908). Tuvo como finalidad formar a niños y jóvenes mapuches como misioneros cristianos, para que ejercieran la evangelización al interior de su familia y comunidad de origen (Muñoz y Quintriqueo, 2019). Ejemplo de esto es la creación del Colegio de Naturales de Chillán (1700), el que fue dirigido a los hijos de logko (principal autoridad política) y ülmen (personas ricas económicamente), estando arraigada en una concepción clasista de la educación, reflejado en su apertura exclusiva a la elite mapuche (Noggler, 1972; Serrano et al., 2013a). Los estudios desarrollados por Serrano et al. (2013a), Gysling (2015), Quintriqueo et al. (2015) y Cano (2018) han constatado que las escuelas misionales cumplieron un rol relevante en la relación entre el pueblo mapuche y Estado chileno posterior a su independencia en 1810, siendo su función: 1) establecer vínculos de confianza con los logkos, para propiciar la estabilidad de los emergentes centros urbanos; y 2) colonizar al pueblo mapuche y establecer el dominio del Estado chileno, enseñando el currículum nacional e imponiendo el castellano como lengua vehicular tanto en la escuela, como en la vida social y familiar. La escuela pública surge mediados del s. XIX, es de carácter laico y se desarrolla bajo la tutela del Estado, ofreciendo una educación gratuita y obligatoria para estudiantes de ambos sexos (Muñoz y Quintriqueo, 2019). Su énfasis fue la conformación de la idea de pueblo chileno y de la identidad nacional común, para propiciar la estabilidad social y política del emergente Estado, invisibilizando a los estudiantes mapuches (Cano, 2018). En La Araucanía, su instalación fue mediante la invasión militar del territorio mapuche autónomo, en el marco de la Guerra de Pacificación (1860-1883), donde en cada ciudad fundada el Estado construyó una escuela (Serrano et al., 2013b). Los internados fueron un dispositivo de control social complementario a las misiones y a la escuela pública, que en La Araucanía separó a los niños mapuches de su familia y comunidad de origen para adoctrinarles disciplinaria e ideológicamente desde los marcos epistémicos de la sociedad hegemónica (Mansilla, Huaiquián y Pozo, 2018). Con este propósito, argumentan Quilaqueo et al. (2014) y Muñoz et al. (2019), el Estado chileno implementó sistemáticamente la exclusión del patrimonio cultural y la invisibilización de los estudiantes mapuches, en base a un ideal de igualdad cultural. Es así como los distintos modelos de escolarización se han complementado en los propósitos de dominación social y religiosa del mapuche, lo que se constata en el incremento del número de escuelas, iglesias católicas y evangélicas en contexto mapuches durante el s. XX (Quintriqueo y Muñoz, 2015; Cano, 2018). En la investigación se sostiene que el reconocimiento de los procesos 
Estudios Pedagógicos XLVII N 1: 391-407, 2021

EDUCACIÓN FAMILIAR E INTERCULTURAL EN CONTEXTO MAPUCHE: HACIA UNA ARTICULACIÓN EDUCATIVA EN PERSPECTIVA DECOLONIAL

históricos asociados a la escolarización permitirá comprender las discontinuidades de la educación familiar mapuche, desde una perspectiva socio-histórica, develando los efectos del colonialismo y la monoculturalidad en la educación escolar.

\section{COLONIALISMO Y MONOCULTURALIDAD EUROCÉNTRICA EN EDUCACIÓN}

El colonialismo y la monoculturalidad eurocéntrica en educación están asociados a un proyecto político diseñado por el Estado, con el fin de homogenizar la población y establecer estructuras jerárquicas, siendo aplicado principalmente desde el sistema escolar formal. En la perspectiva de autores como Kakkar (2017) y Whitehead (2017), el colonialismo es una estrategia política, por medio de la cual se ha construido en las personas indígenas la sumisión, la inferioridad y la vergüenza étnica, por medio de la negación del valor histórico y contemporáneo de sus saberes, lengua vernácula, estructuras sociales, económicas, políticas y culturales. En este proceso, impone la familia nuclear como base de la estructura social para desarticular la reproducción sociocultural indígena (Corntassel y Hardbager, 2019). En cuanto a la monoculturalidad eurocéntrica, Horst y Gitz-Johansen (2010) la definen como un enfoque educativo cuyo fin es eliminar las diferencias étnicas, culturales, lingüísticas y religiosas en contextos caracterizados por la diversidad sociocultural, posicionando la identidad nacional como centro unificador del Estado. En la actualidad responde a los procesos de globalización y la imposición de un ideal universal de sociedad (Conrad, 2018). Por consiguiente, señala Mansilla et al. (2016), el colonialismo y la monoculturalidad eurocéntrica son dispositivos de poder, que han permitido legitimar la dominación ejercida por la sociedad nacional, construyendo un imaginario social, en las personas no indígenas, caracterizado por: 1) el racismo científico, que impone el conocimiento científico occidental como única vía para comprender la realidad, y posiciona a los pueblos indígenas como inferiores desde un punto de vista biológico (Sánchez-Arteaga, Sepúlveda y El-Hani, 2013); 2) la percepción de que los indígenas son personas limitadas intelectualmente, lo que limita sus posibilidades de éxito escolar (Horst et al., 2010; Whitehead, 2017); 3) la exclusión del patrimonio cultural y la memoria histórica indígena del medio escolar, con el propósito de fortalecer la enseñanza disciplinaria del currículum escolar nacional y la aculturación de los estudiantes indígenas (Muñoz, 2016); 4) la negación de la existencia de una episteme de base en el conocimiento indígena y su valor (Quintriqueo y Torres, 2012); y 5) la transmisión de prejuicios sobre la imagen del 'otro' indígena, su tipo de vestimenta y condición social, asignándole una concepción negativa e incluso criminal, que le margina dentro de la sociedad nacional (Aghasaleh, 2018). Esto, en el marco de un sistema educativo marcado social y políticamente por el neoliberalismo, que ha agudizado la contraposición entre el Estado y el pueblo mapuche, centrada en el uso de los recursos naturales, la relación con el medio ambiente y la producción económica, en perjuicio de las familias y comunidades mapuches (Luna, Bolomey y Caniguán, 2018).

Se argumenta en los estudios de Mampaey y Zanoni (2015), Muñoz (2016) y Nahuelpán, Antimil y Lehman, (2019), que, en contexto indígena en general y mapuche en particular, el colonialismo y la monoculturalidad eurocéntrica han conllevado el fracaso escolar generalizado en la población indígena. Esto, producto de una enseñanza que ha avanzado en la dominación social y cognitiva, por sobre el desarrollo del potencial intelectual de 
los estudiantes, propiciado la inclusión social básica del indígena en la sociedad nacional, principalmente en la clase obrera y campesina, como sujetos empobrecidos (Quintriqueo y Muñoz, 2015; Aghasaleh, 2018). Sin embargo, para Horst et al. (2010), estos son factores no considerados en el análisis implementado por los Estados nacionales, quienes asumen como causas del fracaso escolar, los bajos recursos económicos, culturales y lingüísticos de los sujetos, el barrio en que viven y a las familias de pertenencia, ya que estos factores configuran una 'herencia social negativa'. En efecto, lo anterior constituye un conjunto de problemas que condicionan el desarrollo educativo, escolar y social de la población indígena y mapuche en particular, sobre un marco de exclusiones explicitas e implícitas de 'lo mapuche' en el medio escolar, que justifican la 'supremacía blanca occidental' y las asimetrías sociales, instalando la colonialidad del ser, del saber y del poder (Whitehead, 2017; Mansilla et al., 2016). De esta forma, el colonialismo y monoculturalidad eurocéntrica en la escolarización ofrecida por los Estados a los pueblos indígenas han construido desacuerdos epistemológicos con la educación familiar, los que inciden en el fracaso escolar de los estudiantes mapuches.

\section{DESACUERDOS EPISTEMOLÓGICOS QUE INCIDEN EN LA DISCONTINUIDAD DE LA EDUCACIÓN FAMILIAR}

Las discontinuidades educativas se refieren al abandono progresivo de las concepciones de educación, los métodos, prácticas y expectativas educativas respecto de la educación familiar en contexto de diversidad sociocultural, producto de la imposición de la episteme occidental mediante la educación escolar. Lo que, para Novarro, Padawer y Borton (2017) condiciona el éxito o fracaso escolar de los estudiantes pertenecientes a minorías étnico-culturales. Desde la literatura especializada se reconocen las siguientes discontinuidades: 1) el individualismo-colectivismo, que contrapone la noción de aprendizaje autónomo impulsada por la escuela, respecto del sentido de colectividad, colaboración e interdependencia arraigado en la familia y comunidad (Yi, 2018; Yang y Li. 2019); 2) Alfabetización escrita-Tradición oral, donde se establecen relaciones de poder contrapuestas respecto de la base epistemológica de la educación escolar y familiar (Esteban-Guitart et al., 2013; Muñoz, 2016); 3) etnoteorías educativas y parentales, donde se ponen en juego los valores educativos y la representación cultural existente sobre el sujeto que aprende y su posición en un sistema social determinado (Lohmann, Hathcote y Boothe, 2018); 4) pensamiento abstracto-pensamiento práctico e intuitivo, donde la escuela impone un razonamiento hipotético-deductivo, mientras que las familias propician el aprendizaje mediante la experiencia construida (Esteban-Guitart et al., 2013); 5) ecológicas y culturales, donde la dominación desde 'lo europeo occidental' establece barreras tangibles e intangibles que obstaculizan el desarrollo escolar y social de las minorías étnicas-culturales (Ogbu, 1992; Taggart, 2016); 6) modelos y estrategias de enseñanza aprendizaje contrapuestas, donde la familia busca el aprendizaje desde la colaboración, observación, experimentación y espontaneidad, respecto de una escuela centrada en la competencia, la preselección de los contenidos de aprendizajes que quedan ajenos a los intereses de los estudiantes (Bertely, Sartorello y Arcos, 2015); 7) desconocimiento del código, donde las familias y los miembros de la escuela podrían desenvolverse en lenguas distintas o significar los conceptos de distintas formas, así como normas de los procesos 
de educación escolar (Taggart, 2016); y 8) conflicto entre motivos, donde la educación escolar está pensada desde la sociedad hegemónica, deslegitimando el valor y sentido de la educación familiar (Esteban-Guitart et al., 2013).

Estas discontinuidades, han favorecido la minorización de los pueblos indígenas en las sociedades nacionales modernas, mediante la construcción de obstáculos a nivel social, que repercuten negativamente en el desarrollo educativo y escolar de éstos (Ogbu, 1992; Muñoz, 2016). En un plano social, han generado el quiebre en la transmisión del conocimiento propio a las nuevas generaciones. Al respecto, Mekoa (2018) argumenta que los ancianos (poseedores del saber y conocimiento) encuentran barreras de comunicación y de interés con las nuevas generaciones ya escolarizadas, vinculadas a la negación del valor del saber indígena impulsado por la educación escolar. En este contexto, señala Cano (2018) que se han establecido diferencias entre los kimches (sabios) y la elite intelectual mapuche escolarizada, sobre los propósitos de la educación familiar. Estos desacuerdos epistemológicos son factibles de superar mediante la educación intercultural.

\section{EDUCACIÓN INTERCULTURAL EN CONTEXTO INDÍGENA}

La educación intercultural (EI) surge como respuesta al proyecto colonial y monocultural eurocéntrico en educación, con el objetivo de superar los perjuicios que ha conllevado los desacuerdos epistemológicos y la discontinuidad de la educación familiar (Taggart, 2016). Plantea como desafíos responder a la realidad sociocultural de los estudiantes, desprenderse del etnocentrismo y del racismo que han estado a la base de la escolarización (Essomba, 2007), y que han marcado el fracaso del sistema escolar en contexto indígena (Quintriqueo y Muñoz, 2015). Desde una mirada latinoamericana, para Quintriqueo et al. (2015) y Quilaqueo (2018), su propósito es responder a las demandas de los pueblos indígenas respecto del reconocimiento de su patrimonio histórico, en el marco de procesos reivindicativos socioculturales, educativos y territoriales. Busca la contextualización de la educación escolar, incorporando saberes y conocimientos indígenas, para: 1) revitalizar las lenguas indígenas y frenar los procesos de pérdida y/o abandono inducidos por los Estados nacionales; 2) establecer las bases para la revitalización cultural, como respuesta a las políticas asimilacionistas; y 3) propiciar la construcción de aprendizajes escolares contextualizados, significativos y de calidad (Quilaqueo et al., 2014; Quintriqueo et al., 2015). En este proceso, se evita la folclorización de los pueblos indígenas, relevándoles como pueblos vigentes en el presente y aborda los prejuicios y estereotipos históricos y aquellos que emergen en la relación actual con la sociedad dominante (Muñoz, 2016). En la perspectiva de Essomba (2007), el principio en que se sustenta es que, en el establecimiento de una simetría e igualdad de oportunidades entre la sociedad nacional y las minorías étnicas, estos últimos mejorarán sus resultados educativos.

A nivel internacional, existen distintas experiencias sobre educación intercultural en contexto indígena. Destacan casos como el de Nueva Zelanda, quienes consideran de relevanciala aproximación con la sociedad y conocimiento maorí, para establecerinteracciones educativas efectivas (O'Neill, 2017). En el marco del modelo educativo Te kotahitanga se incorpora la importancia de reconocer la identidad sociocultural de los estudiantes, de una cultura académica de altas expectativas y de la colaboración para la construcción de aprendizajes escolares significativos (Bishop y Berriman, 2009). En Australia, desde el año 
2015 se incorporó formalmente en el currículum nacional el patrimonio cultural indígena, siendo considerado una prioridad nacional para el establecimiento de una equidad social y educativa, impactando en los programas de formación de profesores (Mills y Ballantyne, 2016). En Sudáfrica, desde mediados de la década de 1990, se aborda la incorporación del conocimiento indígena al currículum escolar, como sustento para superar los perjuicios del apartheid y el legado colonialista del sistema escolar. Argumentan Meyiwa, Letsekha y Wiebesiek (2013), que esto ha tenido un impacto en la política pública, la cual establece que el currículum escolar debe ser contextualizado a la realidad local, mediante la incorporación del conocimiento indígena de cada pueblo. En Colombia, mediante la EI se incorpora el conocimiento indígena al medio escolar, lo que ha aportado con nuevas fuentes y sustentos pedagógicos para reconstruir las relaciones de poder, descolonizar la memoria y acceder al fondo epistémico del saber y conocimiento indígena (Mavisoy, 2018). Para Tamayo-Osorio (2016), esto ha conllevado abandonar la concepción disciplinaria de la educación, asumiendo una concepción indígena, donde el conocimiento es un todo integral, estableciendo una relación dialógica entre lo indígena y lo escolar, para la formación de niños y jóvenes. En México, las principales experiencias sobre EI son las Secundarias Comunitarias Indígenas, las escuelas autónomas y las milpas educativas. Al respecto, Bertely et al. (2015) precisa que éstas incorporan los saberes y conocimientos indígenas al medio escolar y a la comunidad en la selección de los contenidos curriculares, para la revitalización lingüística-cultural y el diálogo entre el conocimiento indígena y escolar, mediante el Método Inductivo Intercultural. Finalmente, en Chile existe el Programa de Educación Intercultural Bilingüe (PEIB) del Ministerio de Educación (PEIB, 2017), el cual se sustenta en la Ley 19.253 (Chile, 1993), el convenio 169 de la Organización Internacional de Trabajo (OIT, 1989) y la Declaración de las Naciones Unidas sobre los Derechos de los Pueblos Indígenas (Naciones Unidas, 2007). Al amparo de estas normativas, el programa ha buscado revertir los procesos de pérdida y abandono cultural y lingüístico que han vivido los pueblos indígenas producto del carácter colonial de la escolarización implementada por el Estado chileno (PEIB, 2017; Muñoz et al., 2019). Los estudios desarrollados por Muñoz (2016) y Torres (2019), señalan que, en su desarrollo, el PEIB ha buscado la incorporación de contenidos educativos indígenas y la base epistemológica en que estos se sustentan, para aportar al desarrollo educativo y social de los pueblos indígenas. De esta forma, la EI es un proyecto político que busca la equidad del sistema educativo mediante la lucha contra el racismo (Essomba, 2007), y el establecimiento de condiciones para que los estudiantes desarrollen su potencial intelectual, independiente del origen social, cultural, económico y/o religioso (Muñoz, 2016). El desarrollo de la EI tanto a nivel internacional como nacional, por una parte, evidencian la pertinencia, contextualidad y actualidad en tanto campo de estudio. Por otra parte, conforma el marco político y normativo en que se sustenta su implementación en contexto mapuche.

\section{EDUCACIÓN INTERCULTURAL EN CONTEXTO MAPUCHE}

El desarrollo de la EI en contexto mapuche se ha desarrollado desde un modelo de EIB, y desde 1996 ha estado al alero del PEIB. Este programa ha presentado tres etapas, donde la primera (1996-2000) estuvo centrada en la contextualización de la educación en escuelas rurales cercanas a comunidades mapuches. La segunda (2001-2009) buscó el fortalecimiento del bilingüismo y la participación de las comunidades en la gestión 
curricular para la incorporación del conocimiento mapuche en la escuela y la elaboración de Planes y Programas Propios (PPP). En la tercera (2010 a la fecha) se institucionalizó la EIB con su incorporación a la Dirección de Educación General del MINEDUC. Se crea la asignatura de Lengua Indígena, propiciando la revitalización lingüística. Igualmente, se propició la creación de PPP y la formación de los estudiantes para la sana convivencia, el diálogo y el respeto mutuo, desde una perspectiva intercultural (PEIB, 2017). Por consiguiente, el PEIB ha sido una herramienta para el reconocimiento y promoción de la lengua y cultura mapuche, tanto en la política pública educativa, como en el medio escolar.

Desde un plano político, se reconoce el valor que representa la política de EI presentada por el Ministerio de Educación de Chile, la que se desarrolla en el marco de una política educativa neoliberal, donde el sistema escolar está marcado por una lógica de mercado (Paredes, 2016). Así, en primera instancia, es una política que construye un espacio de resistencia al neoliberalismo de su propio marco de referencia, que es la política educativa pública. Sin embargo, las investigaciones de Webb y Radcliffe (2013), Luna (2015), Muñoz (2016) y Torres (2019), han aportado en constatar que la EI en contexto mapuche ha fracasado al posicionarse como un discurso político sin haberse desprendido del colonialismo y la monoculturalidad eurocéntrica de la educación escolar y las prácticas educativas. De esta forma, es una EI funcional al Estado chileno, caracterizándose por: 1) dirigirse de manera exclusiva a la población indígena; 2) centrarse en los aspectos lingüísticos del mapunzugun por sobre los contenidos educativos; 3) contener una visión reductiva de 'lo intercultural', al haber transitado de una propuesta de contextualización transversal del currículum escolar, a su focalización en la asignatura de lengua indígena; 4) ser una educación que reporta bajos niveles de aprendizajes y cobertura curricular, según las evaluación nacional estandarizada SIMCE; 5) marginar los componentes de la educación familiar en el medio escolar; 6) prescindir de la articulación familia, escuela y comunidad en la gestión curricular y en los espacios de toma de decisiones educativas; y 6) reproducir el racismo institucionalizado en la estructura escolar (Muñoz y Quintriqueo, 2019; Torres, 2019). Por lo tanto, la EI se configura como una extensión del colonialismo impulsado desde la política pública educativa hacia el pueblo mapuche, sin buscar el desarrollo del potencial intelectual de los estudiantes mapuches, propiciando la permanencia de las discontinuidades entre la educación familiar y escolar, en el marco del desarrollo de la EI en contexto mapuche. En consecuencia, en la propuesta se sostiene que el carácter funcionalista de la EI implementada ha inducido la extensión de las discontinuidades educativas y sus perjuicios socioculturales, educativos y económicos en contexto mapuche, por lo que se debe avanzar hacia una concepción decolonial de la EI.

\section{HACIA UN PROYECTO DECOLONIAL DE EDUCACIÓN INTERCULTURAL}

La propuesta decolonial de educación intercultural es una construcción política, epistémica y pedagógica que surge desde los pueblos subalternizados, como respuesta al eurocentrismo del conocimiento escolar (Walsh, Fernandes y Candau, 2018). En América Latina, su aplicación ha buscado generar procesos de reconocimiento de 'lo indígena' sobre la base de las diferencias (Viña, Tapia y Walsh, 2010). En ella, el actuar pedagógico implica un posicionamiento político e ideológico respecto de las propuestas estatales de EI, desde la perspectiva de su pertinencia a los procesos de reivindicación de derechos. Para 
autores como Alvarado (2016) y Walsh et al. (2018), busca la liberación de la sociedad, mediante la relativización de la hegemonía del saber y del poder ejercida desde la sociedad hegemónica, valorando las epistemologías indígenas. Así, se establece como una propuesta para la construcción de interacciones centradas en la complementariedad de los pueblos y culturas que componen la sociedad. Argumenta Alvarado (2016), que la implementación de la EI decolonial se sustenta en la participación colectiva de la sociedad en el medio escolar. Además, en la concepción de interculturalidad desde abajo, en la cual son las propias comunidades indígenas, las que proponen, participan de la gestión y de la implementan los proyectos educativos.

En base a la literatura especializada, en la presente investigación se sostiene que avanzar hacia un proyecto decolonial de EI, que supere las discontinuidades educativas en contexto mapuche, es factible considerando los siguientes ejes. Primero, considerar que la educación indígena propicia el acceso al sistema de saberes y conocimientos desde el marco de su propia epistemología, a la cosmovisión y las formas propias de relación del ser humano con el medio social, cultural y espiritual (Luna et al., 2017). Es el reflejo de los mundos de vida en que históricamente se han desarrollado las personas, las formas de conocer y comprender la realidad, de representar el pasado como un marco de referencia para la vida actual y futura en una interacción entre elementos físicos y metafísicos que conforman el mundo mapuche (Nahuelpán et al., 2019; Quintriqueo, Arias-Ortega, Muñoz, Torres, Morales y Peña-Cortés, 2021). En la perspectiva de Corntassel y Hardbarger (2019), esto permitiría establecer procesos educativos que articulan el espacio y conocimiento cultural de las personas, con el contexto de vida actual, posicionando a los pueblos indígenas y sus conocimientos como vigentes en la actualidad. La educación indígena es un lugar de resistencia en contextos de colonización, ya que, a pesar de los procesos de asimilación impulsados por los Estados, las comunidades y familias siguen implementando sus procesos educativos propios, para asegurar la continuidad de su conocimiento y costumbres, siendo un hecho determinante en la búsqueda de la autodeterminación educativa de los pueblos (McKinley y Smith, 2019).

Segundo, incorporar fondos de conocimientos, los que se refieren a contenidos y recursos educativos que toda persona posee producto de su historia social, familiar y laboral, abordando la totalidad de las áreas de desarrollo de las personas y su grupo de pertenencia (Esteban-Guitar et al., 2013). Estos contenidos y recursos educativos están asociados a métodos educativos propios del entorno familiar y social de las personas, por lo que responden a sus propias lógicas de conocimiento, en las que cobran sentido y significado (Brito, Subero y Esteban-Guitart, 2018). Los fondos de conocimiento son una estrategia que emerge para contrarrestar la perspectiva de déficit existente sobre las minorías, en la que se justifica su bajo rendimiento académico (Llopart y Esteban-Guitart, 2016). Es postulado es que, los fondos de conocimiento son factibles de incorporar en el currículum escolar y en las prácticas pedagógicas de los profesores, con el propósito de establecer nexos entre el currículum y la propia experiencia de vida de los estudiantes pertenecientes a minorías (Esteban-Guitart, Llopart y Subero, 2016). En el contexto mapuche, se sostiene que la incorporación al medio escolar de los contenidos y recursos educativos de las familias y la comunidad propicia la contextualización del currículum y el acercamiento de éste a la realidad propia de cada escuela, en un proceso de territorialización de la educación (Quintriqueo et al., 2015; Torres y Friz, 2020). Así, el uso de los fondos de conocimientos de los estudiantes en sus procesos educativos escolares propiciará la construcción de 
aprendizajes significativos y la superación de las discontinuidades educativas (EstebanGuitart et al., 2013; Muñoz, 2016). Esto, al construir los aprendizajes sobre los contenidos educativos escolares en base a los conocimientos y experiencias previas de los estudiantes, construidas en el marco de la educación familiar (Bermudez, Battistón, García y De Longhi, 2017). Por consiguiente, los fondos de conocimientos son un aporte significativo a la construcción de un proyecto decolonial de educación intercultural, al buscar una equidad educativa, la contextualización curricular y la enseñanza del conocimiento de los pueblos indígenas desde sus marcos epistemológicos propios.

Tercero, el pluralismo epistemológico en el cual se reconoce la existencia de múltiples formas de conocer, construir y reconstruir la realidad, los que superan el racionalismo técnico y reductivo de la epistemología del conocimiento científico occidental, reconociendo el valor y pertinencia de las epistemes indígenas (Sánchez-Arteaga, Sepúlveda y El-Hani, 2013). Argumenta Walsh et al. (2018), que el pluralismo epistemológico permite abordar las discontinuidades educativas al contravenir el colonialismo y la monoculturalidad eurocéntrica en los espacios sociales y educativos, contribuyendo en la construcción de una sociedad democrática y diversa. Finalmente, la articulación de la triada familia, escuela y comunidad es central en el establecimiento de un proyecto decolonial de EI, al propiciar el diálogo y negociación de los espacios físicos y simbólicos para la deconstrucción del rol hegemónico de la escuela en contexto mapuche (Corntassel y Hardbarger, 2019; Muñoz et al., 2019). Esto permite la construcción de proyectos conjuntos para el abordaje de las necesidades sociales y educativas en contextos de colonización, como por ejemplo la revitalización lingüística o la enseñanza de oficios tradicionales (Sarivaara y Keskitalo, 2019). Para Lohmann et al. (2018), su articulación centrada en la colaboración incide positivamente a largo plazo en la construcción de aprendizajes escolares. De igual forma, permite resistir los procesos de colonialidad y monoculturalismo eurocéntrico, estableciendo las condiciones para el éxito de la implementación de la EI. Entonces, la articulación de la familia, escuela y comunidad permite la incorporación de la educación indígena y los fondos de conocimiento desde el pluralismo epistemológico en el desarrollo de la EI en perspectiva decolonial en contexto mapuche.

\section{CONCLUSIONES}

La política educativa colonialista y monocultural eurocéntrica diseñada por el Estado chileno ha establecido discontinuidades entre la educación familiar mapuche y escolar, propiciando el establecimiento de desacuerdos epistemológicos que están a la base de la escolarización de los estudiantes mapuches. Así, mediante un rol civilizatorio y nacionalizador, se impuso una educación escolar que negó el valor de toda expresión social, cultural y de conocimiento indígena, para el desarraigo cultural de las nuevas generaciones mapuches. Esto ha conllevado un conjunto de perjuicios educativos, sociales y económicos, que propició la inclusión social básica del mapuche en el Estado chileno, como obreros o campesinos empobrecidos.

Mediante el Programa de Educación Intercultural Bilingüe el Estado buscó responder: 1) a las demandas educativas que las comunidades indígenas realizaron durante el s. XX, buscando el establecimiento de una educación contextualizada y que permitiera revertir los perjuicios de la escolarización monocultural y colonialista; y 2) a los convenios y 
leyes nacionales e internacionales a los que Chile voluntariamente adscribe entre las décadas de 1990 y 2000. No obstante, este programa no reportó los cambios esperados, por el contrario, se configuró como la continuidad y profundización del monoculturalismo eurocéntrico y el colonialismo de la escolarización ofrecida en áreas mapuches. Lo anterior es impulsado por:

- la resistencia de profesores y directivos ante la aplicación del PEIB, argumentando la 'necesidad' de propiciar la enseñanza del currículum nacional homogéneo como estrategia para el logro de la movilidad social ascendente.

- la concepción reductiva de 'lo intercultural' desde la política pública, al ser una educación dirigida a indígenas, centrada principalmente en el bilingüismo y que ha perpetuado la exclusión de la familia y comunidad de la gestión del currículum escolar.

- la continuidad del racismo científico en el medio escolar, que enseña el conocimiento científico como la única forma válida de conocer y comprender la realidad, por lo que es deseable de ser enseñado y aprendido en la escuela.

- profundizar la brecha educativa entre estudiantes mapuches y no mapuches al reportar bajos resultados de aprendizajes del currículum escolar, coartando las posibilidades de desarrollar movilidad social ascendente.

En este contexto, se hace necesario avanzar hacia una propuesta decolonial en educación intercultural que permita abandonar el carácter funcionalista con que ésta se ha desarrollado en las últimas décadas. Esta propuesta, primero, considera la necesidad de establecer un diálogo abierto y constante entre los actores de la familia, escuela y comunidad para una gestión curricular compartida, que propicie la incorporación de componentes de la educación familiar mapuche en la educación escolar. Aquí, es necesario abordar una concepción de 'interculturalidad desde abajo', para revertir las dinámicas de poder ejercidas desde el medio escolar, otorgando una posibilidad concreta a la familia y comunidad de decidir los contenidos, métodos y agentes educativos propios a incorporar en el proceso escolar. Segundo, posiciona como eje central el pluralismo epistemológico, para propiciar una apertura a otras formas de conocer, comprender y explicar la realidad, que supere la estructura del conocimiento científico occidental. De esta forma, se avanzará hacia una educación integral que busque el desarrollo social, cultural educativo y espiritual de las personas. Finalmente, se sostiene que la implementación de una educación intercultural decolonial demanda una nueva forma de comprender la educación en contexto indígena, para abandonar la labor colonial de la escuela y de las relaciones sociales entre indígenas y no indígenas. Esto permitirá avanzar hacia una sociedad más equitativa y cuya educación se centre en el desarrollo del potencial intelectual de los estudiantes, independiente de su origen sociocultural, económico o religioso.

\section{REFERENCIAS BIBLIOGRÁFICAS}

Aghasaleh, R. (2018). Oppressive Curriculum: Sexist, Racist, Classist, and Homophobic Practice of Dress Codes in Schooling. Journal of African American Studies, 22(1), 94-108. https://doi. org/10.1007/s12111-018-9397-5 
Estudios Pedagógicos XLVII Nº 1: 391-407, 2021

EDUCACIÓN FAMILIAR E INTERCULTURAL EN CONTEXTO MAPUCHE: HACIA UNA ARTICULACIÓN EDUCATIVA EN PERSPECTIVA DECOLONIAL

Alvarado, J. (2016), Pensar la universidad en perspectiva decolonial. Revista de Filosofía, 84(3), 116128. Recuperado de https://dialnet.unirioja.es/servlet/articulo?codigo $=6055542$

Ander-Egg, E. (2014). Diccionario de educación. Córdova, Editorial Brujas.

Bermudez, G., Battistón, L., García, M. y De Longhi, A. (2017), Sociocultural Variables That Impact High School Students' Perceptions of Native Fauna: a Study on the Species Component of the Biodiversity Concept. Research in Science Education, 47(1), 203-235. https://link.springer.com/ article/10.1007/s11165-015-9496-4

Bertely, M., Sartorello, S. y Arcos, F. (2015), Vigilancia, cuidado y control étnicopolítico: Red de Educación Inductiva Intercultural. Desacatos, (48), 32-49. Recuperado de http://www.scielo. org. $\mathrm{mx} / \mathrm{pdf} /$ desacatos/n48/n48a3.pdf

Bishop, R. y Berriman, M. (2009). The Te Kotahitanga Effective Teaching Profile. Máori Achievement, set 2, 27-33. Recuperado de https://www.nzcer.org.nz/system/files/journals/set/downloads/ set2009_2_027_0.pdf

Bonfil, G. (1988). La teoría del control cultural en el estudio de procesos étnicos. Anuário Antropológico, (86), 13-53. Recuperado de http://www.ciesas.edu.mx/publicaciones/clasicos/ articulos/TeoriadelControl.pdf

Brito, L., Subero, D. y Esteban-Guitart, M. (2018). Fondos de conocimiento e identidad: Una vía sociocultural de continuidad educativa. Revista Educación, 42(1). Doi: 10.15517/revedu. v42i1.23470

Cano, D. (2018). El rol de la mujer en la educación mapuche. En Serrano, S., Ponce de León, M., Rengifo, F. y Mayorga, R. [Eds.], Historia de la educación en Chile (1810-2010). Tomo III. Democracia, exclusión y crisis (1930-1964) (pp. 303-340). Chile, Taurus.

Chile (1993). Ley 19.253 Establece normas sobre protección, fomento y desarrollo de los indígenas, y crea la Corporación Nacional de Desarrollo Indígena. Recuperado de https://www.leychile. cl/Navegar?idNorma $=30620 \&$ buscar $=19.253$

Conrad, J. (2018). The Big History Project and colonizing knowledges in world history curriculum. Journal of Curriculum Studies. Doi: 10.1080/00220272.2018.1493143

Coña, P. (2017). Lonco Pascual Coña. Testimonio de un cacique Mapuche. Chile, Pehuén.

Corntassel, J. y Hardbarger, T. (2019). Educate to perpetuate: Land-based pedagogies and community resurgence. International Review of Education, 65(1), 87-116. Doi: 10.1007/s11159-018-9759-1

Essomba, M. (2007). Liderar escuelas interculturales e inclusivas. Equipos directivos y profesorado ante la diversidad cultural y la inmigración. Barcelona: Graó.

Esteban-Gitart, M. y Vila, I. (2013). Reflexiones alrededor de las relaciones familia-escuela en contextos de alta diversidad cultural. A modo de introducción. En Esteban-Guitart, M. y Vila, I. [coords.], Experiencias en educación inclusiva: vinculación familia, escuela y comunidad. España, Horsori.

Esteban-Guitart, M., Llopart, M. y Subero, D. (2016). La aproximación de los fondos de conocimiento e identidad. La conexión del currículum y la práctica escolar con los contextos y formas de vida de los aprendices. Papeles de Trabajo sobre Cultura, Educación y Desarrollo Humano, 12(3), 13-19.

Gysling, J. (2015). The historical development of educational assessment in Chile: 1810-2014. Assessment in Education: Principles, Policy y Practice. https://doi.org/10.1080/096959 4X.2015.1046812.

Horst, C. y Gitz-Johansen, T. (2010). Education of ethnic minority children in Denmark: monocultural hegemony and counter positions. Intercultural Education 21(2), 137-151. https:// doi.org/10.1080/14675981003696271.

Kakkar, A. (2017). Education, empire and the heterogeneity of investigative modalities: a reassessment of colonial surveys on indigenous Indian education. Paedagogica Historica. Doi: 10.1080/00309230.2016.1270338.

Lagos, R. (1908). Historia de las misiones del Colegio de Chillán. España: Herederos de Juan Gili. 
Llopart, M. and Esteban-Guitart, M. (2016). Funds of knowledge in 21st century societies: inclusive educational practices for under-represented students. A literature review. Journal of Curriculum Studies, 50(2), 145-161. https://doi.org/10.1080/00220272.2016.1247913

Lohmann, M., Hathcote, A. y Boothe, K. (2018). Adressing the barriers to family-school collaboration: A brief review of the literature and recommendations for practice. International Journal of Early Childhood Special Education, 10(1), 26-32. https://doi.org/10.20489/ intjecse. 454424

Luna, L. (2015). Educación mapuche e interculturalidad: un análisis crítico desde una etnografía escolar. Chungara, Revista de Antropología Chilena 47(4), 659-667. http://dx.doi.org/10.4067/ S0717-73562015005000040.

Luna, L., Bolomey, C. y Caniguan, N. (2018). Educación mapuche en el Chile neoliberal: análisis de tres escuelas dela región de La Araucanía. Sinética. Doi: 10.31391/S2007-7033(2018)0050-007.

Mampaey, J. y Zanoni, P. (2015). Reproducing monocultural education: ethnic majority staff's discursive constructions of monocultural school practices. British Journal of Sociology of Education, 37(7), 928-946. https://doi.org/10.1080/01425692.2014.1001059.

Manquilef, M. (1914). Comentario del pueblo Araucano II. La Jimnasia Nacional (juegos, ejercicios $y$ bailes). Chile, imprenta Barcelona.

Mansilla, J., Huaiquián, C. y Pozo, G. (2018). Infancia mapuche encerrada: internados de las escuelas-misiones en la Araucanía, Chile (1900-1935). Revista Brasileira de Educação, 23. Doi: 10.1590/s1413-24782018230046.

Mansilla, J., Llancavil, D., Mieres, M. y Montanares, E. (2016). Instalación de la escuela monocultural en la Araucanía, 1883-1910: dispositivos de poder y Sociedad Mapuche. Educação e Pesquisa, 42(1), 213-228. http://dx.doi.org/10.1590/S1517-9702201603140562.

Mansilla, J., Ponce De León, M. y Turra, O. (2018). Entre la voluntad intercultural y la voluntad de asimilación: el valor de la lengua mapuche. Misioneros capuchinos bávaros y mapuche en la Araucanía 1896-1929. Opción, 34(87).

Mavisoy, W. (2018). El conocimiento indígena para descolonizar el territorio. La experiencia Kamëntšá (Colombia). Nómadas, (48), 239-248. Doi: 10.30578/nomadas.n48a15.

McKinley, E. and Smith, L. (2019). Towards Self-Determination in Indigenous Education Research: An Introduction. In McKinley, E. and Smith, L. [Eds.] Handbook of Indigenous Education (pp. 1-15). Singapore: Springer Nature Singapore.

Mekoa, I. (2018). Essentialising African indigenous knowledge systems in the midst of globalization and modernity. African Renaissance, 15(1), 11-28. DOI: 10.31920/2516-5305/2018/sin1a1.

Meyiwa, T., Letsekha, T. y Wiebesiek, L. (2013). Masihambisane, lessons learnt using participatory indigenous knowledge research approaches in a school-based collaborative project of the Eastern Cape. South African Journal of Education, 33(4). DOI: 10.15700/201412171329 Recuperado de http://www.sajournalofeducation.co.za/index.php/saje/article/view/845/381.

Mills, C. y Ballantyne, J. (2016). Social Justice and Teacher Education: A Systematic Review of Empirical Work in the Field. Journal of Teacher Education. DOI: 10.1177/0022487116660152.

Muñoz, G. (2016). Participación familiar y comunitaria en el diseño y desarrollo del currículum escolar en contextos de diversidad sociocultural (Tesis doctoral, Universidad Autónoma de Barcelona). Recuperada de https://ddd.uab.cat/record/176053

Muñoz, G. y Quintriqueo, S. (2019). Escolarización socio-histórica en contexto mapuche: implicancias educativas, sociales y culturales en perspectiva intercultural. Educação $e$ Sociedade, 40. http://dx.doi.org/10.1590/es0101-73302019190756.

Muñoz, G., Quintriqueo, S. y Essomba, M. (2019). Participación familiar y comunitaria en la educación intercultural en contexto mapuche. Espacios, 40(19), 21. Recuperado de http://www. revistaespacios.com/a19v40n19/a19v40n19p21.pdf.

Naciones Unidas (2007). Declaración de las Naciones Unidas sobre los Derechos de los Pueblos Indígenas. Naciones Unidas. 
Estudios Pedagógicos XLVII Nº 1: 391-407, 2021

EDUCACIÓN FAMILIAR E INTERCULTURAL EN CONTEXTO MAPUCHE: HACIA UNA ARTICULACIÓN EDUCATIVA EN PERSPECTIVA DECOLONIAL

Nahuelpán, H., Antimil, J. and Lehman, K. (2019). Mapuchezugun Ka Mapuche Kimün: Confronting Colonization in Chile (Nineteen and Twentieth centuries). In McKinley, E. and Smith, L. [Eds.] Handbook of Indigenous Education (pp. 63-83). Singapore: Springer Nature Singapore.

Noggler, A. (1972). Cuatrocientos años de misión entre los araucanos. Chile: Imprenta e Editorial San Francisco.

Novaro, G., Padawer, A. y Borton, L. (2017). Interculturalidad y educación argentina desde una perspectiva comparativa. Educação \& Realidade, 42(3), 939-958. http://dx.doi.org/10.1590/2175623672321 .

O'Neill, J. (2017). A biographical experience of teacher education in Aotearoa New Zealand", European Journal of Teacher Education. DOI: 10.1080/02619768.2017.1385057

Ogbu, John (1992), "Understanding Cultural Diversity and Learning", Educational Resaercher 21(8), 5-24. Recuperado de https://pdfs.semanticscholar.org/f930/570df8ecb7cfd2d474b97e6b db52f1db925a.pdf

Organización Internacional del Trabajo [OIT] (1989). Convenio 169 de la Organización Internacional del Trabajo sobre pueblos indígenas y tribales en países independientes de la Organización Internacional del Trabajo. Recuperado de https://www.leychile.cl/Navegar?idNorma=279441 \&buscar=convenio+169

Paredes, R. (2016). El sistema de vouchers en la educación chilena. En Corvalán, J., Carrasco, A. y García-Huidobro, J. [Eds.] Mercado Escolar y Oportunidad Educacional. Libertad, diversidad $y$ desigualdad (57-80). Chile, Ediciones UC.

Programa de Educación Intercultural Bilingüe [PEIB] (2017). Programa de Educación Intercultural Bilingüe (2010-2016). Chile: Ministerio de Educación.

Quilaqueo, D. (2005). Educación Intercultural desde la Teoría del Control Cultural en Contexto de Diversidad Sociocultural Mapuche. Cuadernos Interculturales, 3(4), 37-50. Recuperado de https://www.redalyc.org/pdf/552/55200404.pdf.

(2018). Conocimientos sociales y culturales para una educación escolar en contexto mapuche. Opción, 34(87).

Quilaqueo, D. y Quintriqueo, S. (2017). Métodos educativos mapuches: retos de la doble racionalidad educativa. Aportes para un enfoque educativo intercultural. Chile: Universidad Católica de Temuco.

Quilaqueo, D., Fernández, A. y Quintriqueo, S. (2017). Tipos discursivos a la base de la educación familiar mapuche. Universum, 32(1), 159-173. Recuperado de https://scielo.conicyt.cl/pdf/ universum/v32n1/0718-2376-universum-32-01-00159.pdf

Quilaqueo, D., Quintriqueo, S., Torres, H. y Muñoz, G. (2014). Saberes educativos mapuches: aportes epistémicos para un enfoque de educación intercultural. Chungará, 271-283. DOI: http://dx.doi. org/10.4067/S0717-73562014000200008

Quintriqueo, S. y Muñoz, Gerardo (2015). Demandes éducationnelles sociohistoriques et éducation interculturelle en contexte mapuche. En Salas, R. y Le Bonniec, F [Eds.], LES MAPUCHE À LA MODE: Modes d'existence et de résistance au Chili, en Argentine et au-delà (95-122). Francia: L'Harmattan.

Quintriqueo, S. y Torres, H. (2012). Distancia entre el conocimiento mapuche y el conocimiento escolar en contexto mapuche. Revista Electrónica de Investigación Educativa, 14(1), 16-33. Recuperado de http://redie.uabc.mx/vol14no1/contenido-quintriqueotorres.html

Quintriqueo, S., Arias-Ortega, K., Muñoz, G., Torres, H., Morales, S. y Peña-Cortés, F. (2021). Conocimientos geográficos y territoriales con base epistémica en la memoria social mapuche. Revista Brasileira de Ciências Sociais, 36(106), e3610603. DOI: 10.1590/3610603/2021 (en prensa).

Quintriqueo, S., Quilaqueo, D., Peña-Cortés, F. y Muñoz, G. (2015). Conocimientos culturales como contenidos de la educación familiar mapuche. Alpha, 40, 131-146 DOI: http://dx.doi. org/10.4067/S0718-22012015000100010 
Sánchez-Arteaga, J., Sepúlveda, C. y El-Hani, C. (2013). Racismo científico, procesos de alteración y enseñanza de ciencias. Magis, Revista Internacional de Investigación en Educación 6(12), 5567. Recuperado de https://www.redalyc.org/pdf/2810/281029756004.pdf

Sarivaara, E. and Keskitalo, P. (2019). Sámi Language for All: Transformed Futures Through Mediative Education. In McKinley, E. and Smith, L. [Eds.] Handbook of Indigenous Education (467-482). Singapore: Springer Nature Singapore.

Serrano, S., Ponce De León, M. y Rengifo, F. (2013a). Historia de la Educación en Chile (18102010). Tomo I Aprender a leer y escribir (1810-1980). Chile, Taurus.

(2013b). Historia de la Educación en Chile (1810-2010). Tomo II La educación nacional (1880-1930). Chile, Taurus.

Taggart, A. (2016). The Role of Cultural Discontinuity in the Academic Outcomes of Latina/o High School Students. Education and Urban Society. DOI: 10.1177/0013124516658522

Tamayo-Osorio, C. (2016). Currículo escolar, conocimiento [matemático] y prácticas sociales: posibilidades otras en una comunidad indígena Gunadule. Educação e Pesquisa, 42(1), 903919. DOI: http://dx.doi.org/10.1590/s1517-9702201612145827

Torres, H. (2019). Análisis de la educación intercultural desde una experiencia etnográfica en contexto mapuche. En Quintriqueo, S. y Quilaqueo, D. [Coords.], Educación e Interculturalidad: aproximación crítica y decolonial en contexto indígena (pp. 287-301). Chile: Universidad Católica de Temuco.

Torres, H. y Friz, M. (2020). Elementos críticos de la escuela en territorio mapuche. Educar em revista, 36, e66108. https://doi.org/10.1590/0104-4060.66108

Viña, J., Tapia, L. y Walsh, C. (2010). Construyendo Interculturalidad Crítica. Bolivia: Instituto Internacional de Integración del Convenio Andrés Bello.

Walsh, C., Fernandes, L. y Candau, V. (2018). Colonialidade e pedagogia decolonial: Para pensar uma educação outra. Arquivos Analíticos de Políticas educativas 26(83). DOI: https://doi. org/10.14507/epaa.26.3874

Webb, A. y Radcliffe, S. (2013). Mapuche Demands during Educational Reform, the Penguin Revolution and the Chilean Winter of Discontent. Studies in Ethnicity and Nationalism, 13(3), 319-341. DOI: 10.1111/sena.12046

Whitehead, K. (2017). British teachers' transnational work within and beyond the British Empire after the Second World War. History of Education, 46(3), 324-342. DOI: https://doi.org/10.108 0/0046760X.2016.1268214

Yang, W. y Li, H. (2019). Changing culture, changing curriculum: a case study of early childhood curriculum innovations in two Chinese kindergartens. The Curriculum Journal 30(3), 279-297. DOI: https://doi.org/10.1080/09585176.2019.1568269

Yi, Jung-Soo (2018). Revisiting individualism-collectivism: A cross-cultural comparison among college students in four countries. Journal of Intercultural Communication, 47. Recuperado de https://www.immi.se/intercultural/nr47/jung.html 
